\title{
An Improved Force Model for Orthogonal Micro- Milling based on Strain Gradient Plasticity Theory
}

\author{
Jihua $\mathrm{Wu}$ \\ School of Mechanical Engineering, Shaoxing University \\ Shaoxing, P.R. China, 0575-88341528 \\ wujihua@usx.edu.cn
}

\begin{abstract}
Micro-milling is emerging as a promising fabrication process to serve the increasing needs in making miniature parts with complex three-dimensional $3 D$ features. The prediction model of cutting force is important in a correct choice of cutting parameters, reasonable design of micro tools and accurate estimation of machining tolerances. The model should be required the capability to illustrate material behavior when the uncut chip thickness reduces to the micro scale. To answer this need, the paper proposed a cutting force model in orthogonal micro milling based on strain gradient plasticity theory. A set of slot milling experiments with a straight cutting edge were conducted to verify the proposed model. The results show a good agreement between the predicted and measured force components.
\end{abstract}

Keywords- strain gradient; micro-milling; cutting force; dislocation density

\section{INTRODUCTION}

Micro-milling is emerging as a promising fabrication process to serve the increasing needs in making miniature parts with complex three-dimensional 3D features to high relative accuracies in the fields that include optics, electronics, medicine, biotechnology, and avionics. Reliable cutting force model of micro milling is indispensable for better understanding the process parameters and effectively control the process outputs such as surface error, vibration and tool wear.

Analytical method have been favored for modeling forces in metal cutting because they are easy to implement and can give much more insight about the physical behavior in metal cutting compared with laborious FEM method. Dornfeld et al. reviewed the prediction models of cutting forces at micro scale ${ }^{[1]}$. Cutting force prediction in micro scale machining is complicated by a number of issues that are fundamentally different from macro scale machining and influence the underlying mechanism to the process. The cutting force models in the literatures have illustrated the effect of workpiece ${ }^{[2]}$, tool condition $^{[3]}$ and tool-workpiece contact ${ }^{[4]}$, which given an impulse of the prediction mechanics of micro-cutting. However, recent experiments have repeatedly shown that strong size effect in micro-cutting ${ }^{[3]}$, few developed models can present the material behavior in the micro scale analytically and describe the characteristic size effect phenomenon in the micromachining.

Conventional cutting mechanics developed based on

This technique is the subject of Zhejiang province education department(Y201225873) classical theory of plasticity, but classical theory of plasticity posses no internal length scale and is hard to explain the size effect in the micro-cutting process, which has motivated the application of strain gradient plasticity theory in micro-cutting. Laheurte et al. quantified energy parameters involved in the cutting process based on the strain gradient theory with couple stresses $^{[5]}$. Joshi modeled the size-effect in the parallel-sided primary deformation zone in machining using strain gradient plasticity theory ${ }^{[6]}$. Liu developed a coupled thermo-mechanical finite element model that is capable of accurately predict the size effect in specific cutting energy formulation incorporating strain gradient plasticity to simulate the orthogonal microcutting process ${ }^{[7]}$.

This paper aims to develop a cutting forces model to incorporate the size effect based on the strain gradient plasticity theory and performs a series of orthogonal micro-milling experiments to verify the proposed model.

\section{FORCE MODEL DEVELOPMENT}

\section{A. Evaluation of non local stress, strain based on strain gradient plasticity theory.}

Materials show different features of a micro-scale deformation process from which of macro scale. Recent experiments have repeatedly reported the presence of strong size effect. A number of researchers have modeled the sizeeffect in a deformation process using the strain gradient plasticity theory. Eringen considered a higher-order extension of the local plasticity theory to describe the strain gradient formulation ${ }^{[8]}$. Non-local variables are described as a weighted average local variable on a neighborhood union condition. The analysis model of micro-cutting processes can be shown as Fig. 1.

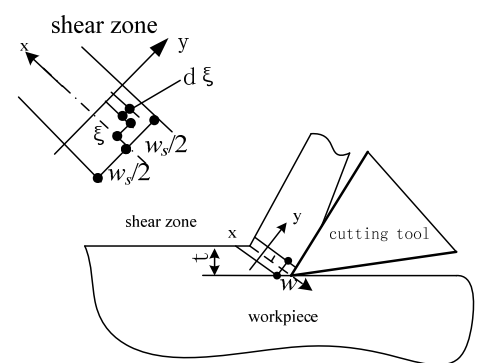

Figure 1 Analysis model of shear zone in the micro-cutting processes 
As is shown in Fig.1, $w_{s}$ is the width of the parallel shear zone. The origin of coordinate is located at the center of a shear zone. The average shear strain in the primary shear zone is expressed using the following equation:

$$
\gamma=\frac{1}{\Omega} \int_{-t / 2}^{t / 2} g(\xi) \gamma(y+\xi) d \xi
$$

$\zeta$ is the distance form point $y$ to any point of its neighborhood, $\xi \in\left[-\frac{w_{s}}{2}, \frac{w_{s}}{2}\right]$. And $g(\xi)$ can be given as gauss distribution in order to make non local property decreased with an increase of the width of the shear zone $w$ :

$$
g(\zeta)=e^{-\frac{\zeta^{2}}{4 l^{2}}}
$$

$\Omega$ is the integral of weighting function.

$$
\Omega=\int_{-\frac{w}{2}}^{\frac{w}{2}} g(\zeta) d \zeta
$$

Taylor series expansion is employed to impress non local shear strain can be given by:

$$
\gamma(y+\zeta)=\gamma(y)+\frac{d \gamma(y)}{d y} \zeta+\frac{1}{2 !} \frac{d^{2} \gamma(y)}{d y^{2}} \zeta^{2}+\ldots .
$$

Substituting (2) into (1), the nonlocal shear strain is shown as:

$$
\gamma=\frac{1}{\Omega} \int_{-t / 2}^{t / 2} g(\xi) \gamma(y) d \xi+\frac{1}{2 \Omega} \int_{-t / 2}^{t / 2} g(\xi) \gamma(y+\xi) d \xi
$$

So non local effective flow stress $\sigma$ can be calculated by:

$$
\sigma=C \varepsilon^{n}
$$

where $n$ is harden coefficient, and C is experiment constant.

Suppose the total strain is shear strain, and shear flow stresses can be given by effective stress.

$$
\sigma=\sqrt{3} \tau
$$

The non local shear stress is given by:

$$
\tau=\frac{\sqrt{3}}{3} \gamma^{n}
$$

\section{CUTTING FORCE MODEL OF ORTHOGONAL MICRO- MILLING}

The following assumptions are made to the proposed force model.

(1) The orthogonal micro-milling operation is assumed to be chatter free.

(2) The tool is rigid.
The principal force using Merchant's force expression is given by ${ }^{[9]}$ :

$$
d F_{c}=\frac{\tau w \cos \left(\beta-\gamma_{e}\right)}{\sin \phi \cos \left(\phi+\beta-\gamma_{e}\right)} d t
$$

where $w$ is width of cut, $\beta$ is the friction angle, $\phi$ is the shear angle, $\gamma_{e}$ is the effective rake angle.

$$
d F_{t}=\frac{\tau w \sin \left(\beta-\gamma_{e}\right)}{\sin \phi \cos (\phi+\beta-\gamma)} d t
$$

The instantaneous undeformed chip thickness in orthogonal micro -milling can be found in Eq.(12).

$$
t=R+r_{e}+f \sin \theta-\sqrt{\left(R+r_{e}\right)^{2}-f^{2} \cos ^{2} \theta}
$$

where $f$ is the feed rate per tooth. $\theta$ is the tool ration angle.

So the differential undeformed chip thickness is expressed as the following:

$$
d t=f \cos \theta-1 / 2 f^{2} \sin 2 \theta\left(\left(R+r_{e}\right)^{2}-f^{2} \cos ^{2} \theta\right)^{-1 / 2} d \theta
$$

Because the cutting edge radius is ignored. Substituting Eq.(6)and Eq.(13) into Eq (9), Eq.(12) into Eq(8) and Eq(9), and after rearrangement as:

$$
d F_{c}=\frac{\tau_{0} w \cos \left(\beta-\gamma_{e}\right)}{\sqrt{1+2 \pi r_{e} \arccos \left(\frac{f}{r_{e}} \sin \theta-\sqrt{1-\left(\frac{f}{r_{e}}\right)^{2} \cos ^{2} \theta}\right)}}\left(f \cos \theta-1 / 2 f^{2} \sin 2 \theta\left(R^{2}-f^{2} \cos ^{2} \theta\right)^{-1 / 2}\right) d \theta
$$

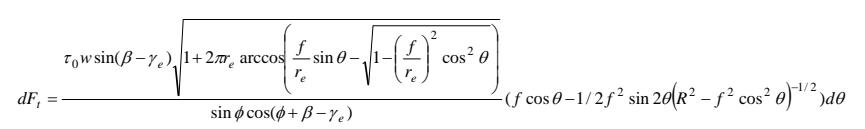

Taking integration, the expressions for the cutting force model were derived as:

$$
\begin{gathered}
F_{c}=\int_{\theta_{s}}^{\theta_{e}} \frac{\tau_{0} w \cos \left(\beta-\gamma_{e}\right) \sqrt{1+2 \pi r_{e} \arccos \left(\frac{f}{r_{e}} \sin \theta-\sqrt{1-\left(\frac{f}{r_{e}}\right)^{2} \cos ^{2} \theta}\right)}}{\sin \phi \cos \left(\phi+\beta-\gamma_{e}\right)} \\
\quad *\left(f \cos \theta-1 / 2 f^{2} \sin 2 \theta\left(R^{2}-f^{2} \cos ^{2} \theta\right)^{-1 / 2}\right) d \theta
\end{gathered}
$$

$$
\begin{aligned}
F_{c}=\int_{\theta_{s}}^{\theta_{e}} \frac{\tau_{0} w \sin \left(\beta-\gamma_{e}\right) \sqrt{1+2 \pi r_{e} \arccos \left(\frac{f}{r_{e}} \sin \theta-\sqrt{1-\left(\frac{f}{r_{e}}\right)^{2} \cos ^{2} \theta}\right.}}{\sin \phi \cos \left(\phi+\beta-\gamma_{e}\right)} \\
\quad *\left(f \cos \theta-1 / 2 f^{2} \sin 2 \theta\left(R^{2}-f^{2} \cos ^{2} \theta\right)^{-1 / 2}\right) d \theta
\end{aligned}
$$


where $\theta_{s}$ is the integrating start angle, $\theta_{e}$ is the integrating end angle. The friction angle $\beta$ and shear angle can be calculated according to the literature ${ }^{[9]}$.

The effective rake angle $\gamma_{e}$ in the micro-cutting processes is given by:

$$
\gamma_{e}=\left\{\begin{array}{cc}
\sin ^{-1}\left(\frac{t}{r_{e}}-1\right) & t \leq r_{e} \\
\gamma_{n} & t>r_{e}
\end{array}\right.
$$
Fig.2.

The verified orthogonal micro-cutting operation is shown in

The cutting forces of feed and normal directions which can be measured directly in the experiments are expressed as:

$$
\begin{aligned}
& F_{x}=F_{\mathrm{t}} \cos \theta+F_{r} \sin \theta \\
& F_{y}=F_{\mathrm{t}} \sin \theta-F_{r} \cos \theta
\end{aligned}
$$

\section{EXPERIMENTAL SET-UP}

For the verification of the model performance, a set of slot milling operations using a single insert indexable cutting tool with a straight cutting edge (helix angle $=0$ ) was undertaken on an ACE-V500 ultra-precision milling machine. The workpiece is mounted upon the force platform (Kistler Type 9275A), which is connected with a plate fixture to acquire force data in $\mathrm{X}$ and $\mathrm{Y}$ axes. The charge amplifier of the force platform was connected to a PC-Pentium data acquisition system as shown in Fig.2.

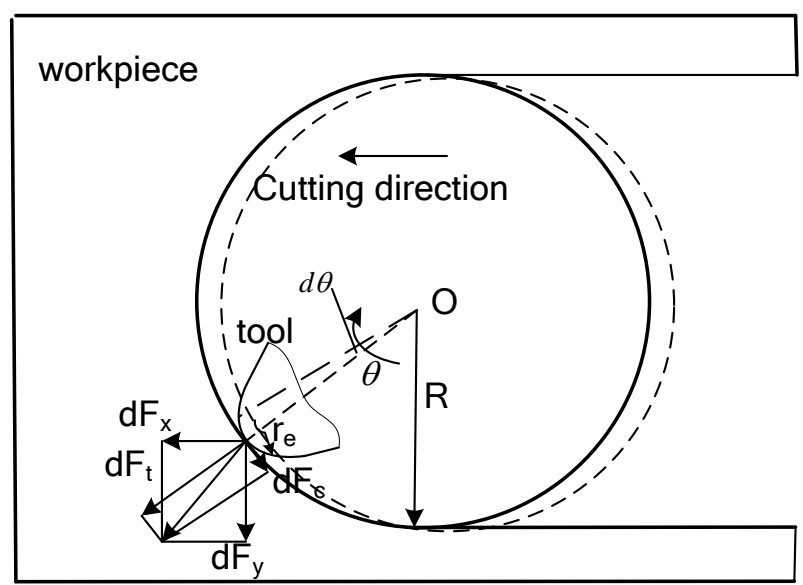

Figure 2 Cutting forces developed during slot micro-milling

The workpiece material used was AISI 1045 (pre-hardened at $30 \mathrm{HRC}$ ). The cutting tool material was uncoated tungsten carbide (WC). In this operation, the bottom of the insert stays in the air and does not engage with the workpiece, only the side cutting edge of the tool was engaged with thin workpiece plates. Therefore, chip flow with plane strain deformation in a $x-y$ plane was obtained. The cutting conditions (feed per tooth and

\begin{tabular}{|c|c|}
\hline \multicolumn{2}{|c|}{ Tool (uncoated tungsten carbide WC) } \\
\hline Tool geometry & $\begin{array}{c}\text { Rake angle } 0^{\circ} \text {, clearance } \\
\text { angle } 10^{\circ}\end{array}$ \\
\hline edge radius $[\mu m$ ] & 16 \\
\hline tool radius $[\mathrm{mm}]$ & 25 \\
\hline \multicolumn{2}{|c|}{ Workpiece properties (AISI 1045) } \\
\hline Intrinsic length scale [ $\mu \mathrm{m}$ ] & 27.6 \\
\hline Shear modulus $G$ [GPa] & 80 \\
\hline \multicolumn{2}{|c|}{ Orthogonal cutting condition } \\
\hline Cutting speed [m/s] & $5,10 、 15,20$ \\
\hline $\begin{array}{l}\text { Undeformed chip thickness } \\
\text { [ } \mu \mathrm{m}]\end{array}$ & $1,4 、 8 、 10$ \\
\hline Depth of cut [mm] & 1 \\
\hline
\end{tabular}
cutting speed) and related milling parameters used during the experiments are given in table 1.

\section{RESULTS AND DISCUSSIONS}

The flow chart of the proposed model to obtain the cutting force and thrust force in the micro-cutting is illustrated in Fig.3.

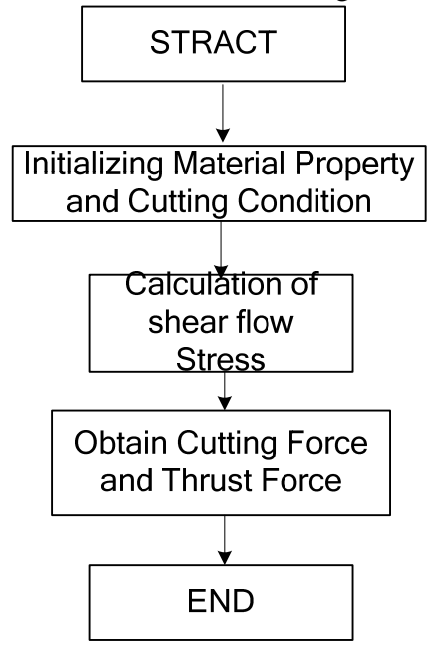

Figure3 Flowchart of the proposed cutting forces model

The cutting forces Fx and Fy of predicted theoretically are compared with the cutting forces obtained during the experiment at feed rate of $4 \mu \mathrm{m} / \mathrm{r}$, velocity at $10 \mathrm{~m} / \mathrm{s}$ as shown in Fig.4.

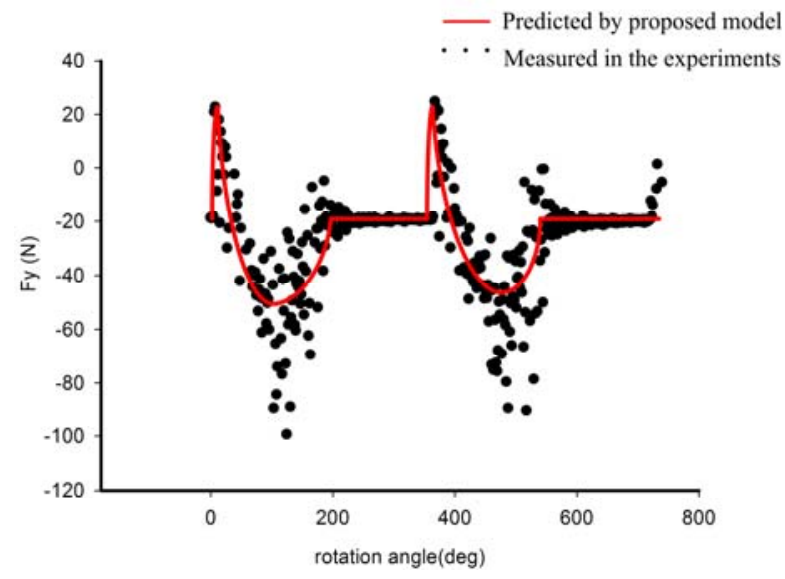




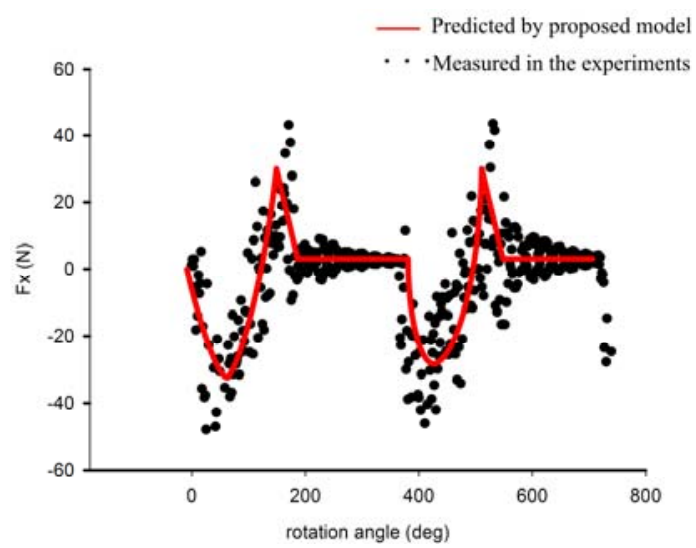

Figure 4 Comparison of predicted and experimental force at feed rate $4 \mu \mathrm{m} / \mathrm{r}$

It is seen that the cutting force changes with changing the rotation angle periodically. The error rate in the radial direction is lower than $13 \%$ while that in the tangential direction indicated $10-15 \%$ in terms of the maximum cutting forces, as is shown in table 2.

TABLE 2 ERROR BETWEEN PREDICTED AND EXPERIMENTAL MAXIMUM CUTTING FORCES

\begin{tabular}{ccc}
\hline $\begin{array}{c}\text { Feed } \\
{[\mu \mathrm{m} / r \mathrm{r}]}\end{array}$ & $\begin{array}{c}\text { Error of maximum } \\
\text { thrust force [\%] }\end{array}$ & $\begin{array}{c}\text { Error of maximum } \\
\text { cutting force[\%] }\end{array}$ \\
\hline 1 & 10.3 & 12.3 \\
4 & 9.1 & 10.7 \\
8 & 10.6 & 14.1 \\
10 & 12.2 & 12.9 \\
\hline
\end{tabular}

The proposed model is verified to show a good agreement between the predicted and measured force components.

\section{SUMMERY}

The paper has proposed a cutting force model in orthogonal micro milling based on strain gradient plasticity theory to represent the effect of material behavior and the tool edge radius on the forces at micro scale. A set of slot milling experiments using a single insert indexable cutting tool with a straight cutting edge were conducted to verify the proposed model. The good correlation of the predicted cutting force with the experimental force indicates that strain gradient plasticity theory can illustrate the material behavior and the effect of the tool edge radius in the orthogonal micro-milling.

\section{REFERENCES}

[1] Dornfeld, D., Min, S., Takeuchi, Y., "Recent Advances in Mechanical Micromachining.” Ann CIRP. vol. 55(2), pp.745-768,2006.

[2] Kim, J.D., Kim D.S., “Theoretical analysis of micro-cutting characteristics in ultra-precision machining.” J. Mater. Process. Technol. Vol. 49, pp. 387-398. 1995

[3] Liu, X, Jun, M.B.G, DeVor, R.E, Kapoor, S.G., "Cutting Mechanisms and Their Influence on Dynamic Forces, Vibrations and Stability in Micro-Endmilling.” Proceedings of IMECE, Anaheim, California, pp.13-20. 2004.

[4] Kang, I.S., Kim, J.S., Kim, J.H. etal., “A mechanistic model of cutting force in the micro end milling process.” J. Mater. Process. Technol. Vol.187-188, pp.250-255. 2007.

[5] Laheurte R, Cahuc O, Darnis P, et al., "Behaviour law for cutting process.” The Int J Adv Manuf Tech. vol.29, pp.17-23. 2006.

[6] Joshi, S.S., Melkote S.N., "An explanation for the size-effect in machining using strain gradient plasticity.” J Manu Sci E-T ASME. Vol.126,pp. 679-685. 2004.

[7] Liu K., Melkote, S. N., "Material Strengthening Mechanisms and Their Contribution to Size Effect in Micro-Cutting.” J Manu Sci E-T ASME. Vol.128, pp.731-740. 2006.

[8] A. Cemal Eringen, "Electromagnetic theory of microstretch elasticity and bone modeling J. Eng. Sci vol. 42 pp. 231-2422004

[9] Merchant M E., "Mechanics of the metal cutting process. Orthogonal cutting and type 2 chip.” J Appl Phys. Vol.16(5), pp.267-276. 1945. 